\title{
Experimental Study into Effect of Developing Section Length on The Heat Transfer Process in a Horizontal Annulus
}

\author{
Dr. Akeel A. Mohammed \\ University of Technology \\ Mechanical Eng. Dep.
}

\author{
Ameer A. Jadoaa, Assistant lecturer. \\ University of Technology \\ Electromechanical Eng. Dep. \\ Email: Ameeer_79@yahoo.com
}

\begin{abstract}
An experimental investigation has been presented to study the heat transfer process in thermally developed region of horizontal concentric annulus with an adiabatic inner tube and uniformly heated outer tube. Three lengths of entrance section have been used to achieve the hydrodynamically fully developed flow before test section with $\left(L / D_{h}=50,75\right.$ and 100$)$. The study has covered the range of Reynolds number (450 $\leq$ $\mathrm{Re} \leq \mathbf{2 0 0 0})$ and Richarson number $(0.1 \leq \mathrm{Ri} \leq \mathbf{0 . 7})$. Results show that the heat transfer process in the lower part of annulus is better than that in the upper part; and the vortex strength increases as Rayligh and Rynolds numbers increase. An empirical correlation for the average Nusselt number as a function of Rayliegh and Reynolds numbers and $\mathrm{L} / \mathrm{D}_{\mathrm{h}}$ has been deduced and compared with available literatures to give the same trend and behavior.
\end{abstract}

Key wards: Developing section (calming), Heat transfer, Annulus.

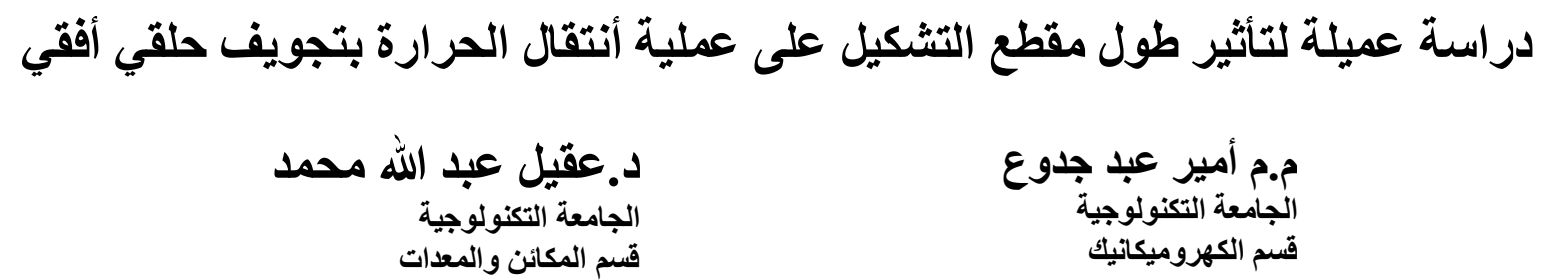

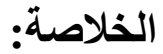

تم أجراء تجارب عملية لاراسة عملية أنتقال الحرارة بمنطقة التثكيل الحراري لتجويف حلقي

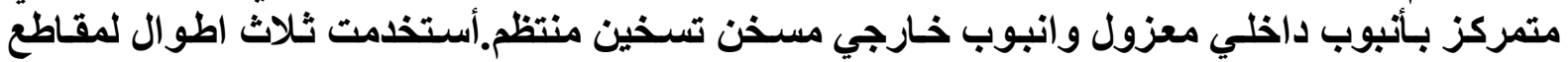

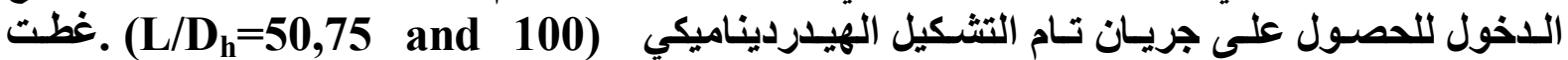

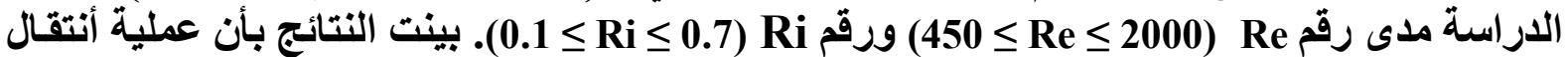

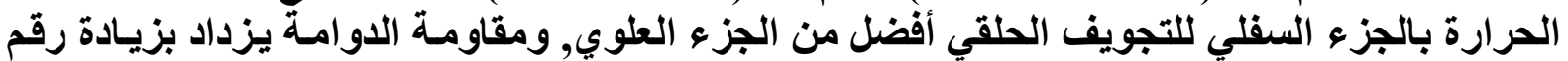

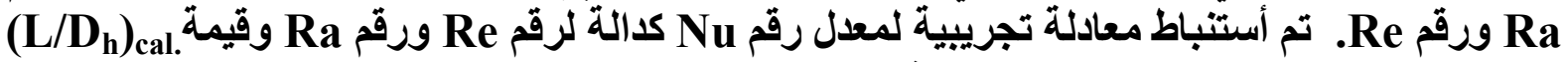
لمقطع المخمد وقورنت مع الادبيات السابقة لتعطي نفس السلوك ولمع والمنحى.
} 


\section{AINTRODUCTION}

The influence of free convection on forced convective heat transfer can be significant. In many practical situations where the buoyancy force is high and the forced flow is low, the free and forced convection effects can be of comparable order of magnitude. This occurs in heat exchangers designed for viscous liquids, pipelines used for transporting oil, and heat exchangers for gas floes [Yousef and Tarasuk 1982]. When heat is transferred through the outer tube wall of a horizontal annulus, the warmer fluid moves upward along the side walls, and, by continuity, the heavier fluid near the center gap of annular duct flows downward. As a result, a two symmetrical spiral-like motion is formed. This secondary flow is driven by the radial temperature variation. Experimental and theoretical studies on combined free and forced convection in tubes, channels, and around immersed bodies have been reported by several investigators. A few investigations deal with the influence of free convection on forced convection in a horizontal annuli (Ciampi, et al 1987, Habib, et al 2001, Kaviany 1986, Kotake, et al 1985, Mohammed 2007, Nazrul,et al 2001, Nieckele, et al 1985, Gada A. Sadiq 2009). No experimental data, about the influence of using of calming section with different values of $\left(\mathrm{L} / \mathrm{D}_{\mathrm{h}}\right)$ before the heated section of annulus with an adiabatic inner tube and uniformly heated outer tube, are available.

The primary motivation of the problem considered in the present study is to improve our understanding of the combined convection phenomena under various effected variables to enhance finally the heat transfer coefficients whenever this field is used in the practical applications. The important objective of this study can be recorded as follows:

1- To present the effect of increasing of calming section length (i.e; the values $L / D_{h}$ ) on the heat transfer process.

2- To know at any angular location and longitudinal position, the heat transfer coefficient becomes maximum.

3- To present the effect of $\mathrm{Ra}, \mathrm{Re}, \mathrm{q}$ and $\mathrm{Ri}$ on the heat transfer process.

4- Finally to obtain a correlation describes the average Nusselt as a function of $\mathrm{Ra}, \mathrm{Re}$, and $\mathrm{L} / \mathrm{D}_{\mathrm{h}}$; and compare it with available literature.

\section{EXPERIMENTAL APPARATUS}

\section{Flow Loop:}

The test facility used in this experimental investigation is shown in Fig (1). Air used as the working fluid was circulated around the loop using centrifugal fan (1). The air flow rate through the test section was regulated using flow control valve (14). The test section was mounted on a rigid wooden board. Three thermocouples were used to measure the inlet and outlet bulk temperature (15), one was fixed in mixing chamber, and the two others were fixed in the exit Teflon piece. The flow rate was measured by using $\mathrm{H}_{2} \mathrm{O}$ manometer (3).

\section{Test section and heating element:}

The annular tube test section (10) was constructed using copper and aluminum for inner and outer tube; respectively, with length of $1200 \mathrm{~mm}$, inner diameter of outer of $21.9 \mathrm{~mm}$, and outside diameter of inner tube of $52.3 \mathrm{~mm}$. The experimental apparatus consisted of three parts: a hydrodynamic developing length (8) of $1500 \mathrm{~mm}, 2250 \mathrm{~mm}$ and $3000 \mathrm{~mm}$, a mixing chamber (5) length of $300 \mathrm{~mm}$, a heated section (10) length of $1200 \mathrm{~mm}$. the developing section was constructed with the heated section by Teflon connection pieces (9) bored with the same inside diameter of the heated outer tube and outside diameter of the inner 
tube which was insulated from its inside by fiber glass. The Teflon material was chosen because of its low thermal conductivity so the test section was first covered by an electrical insulating as shown in Fig.(1) varnish coating and then wrapped by a layer of asbestos with thickness of $1.5 \mathrm{~mm}$ to protect the varnish from the heater wires. The heating wires were then covered with high temperature; high thermal conductivity cements to ensure that the wires remained firmly in place at all operating temperatures. Also to uniformly distribute the input heat. Layers of asbestos (12) and Gibson (13) covered the whole test section with thickness of $6.5 \mathrm{~mm}$ and $5.7 \mathrm{~mm}$, respectively for thermal insulation as shown in Fig.1. Two pairs of thermocouple were installed in asbestos layer between the heated and the insulation as two thermocouples at three stations along the heated section to perform heat loss calculation through the test section lagging and the measured heat loss was used in adjusting the electrical power input. Also, to evaluate the heat losses from the ends of the test section, two thermocouples were fixed in each Teflon piece. By knowing the distance between these thermocouples and the thermal conductivity of the Teflon, the end losses can be calculated. Wall temperatures were measured within the heated section by thermocouples type $(\mathrm{k})$. The inlet bulk temperature was measured at the settling chamber and the outlet bulk temperature was measured by inserting two thermocouples in mixing chamber. A straight line was fitted between the inlet and outlet bulk temperatures.

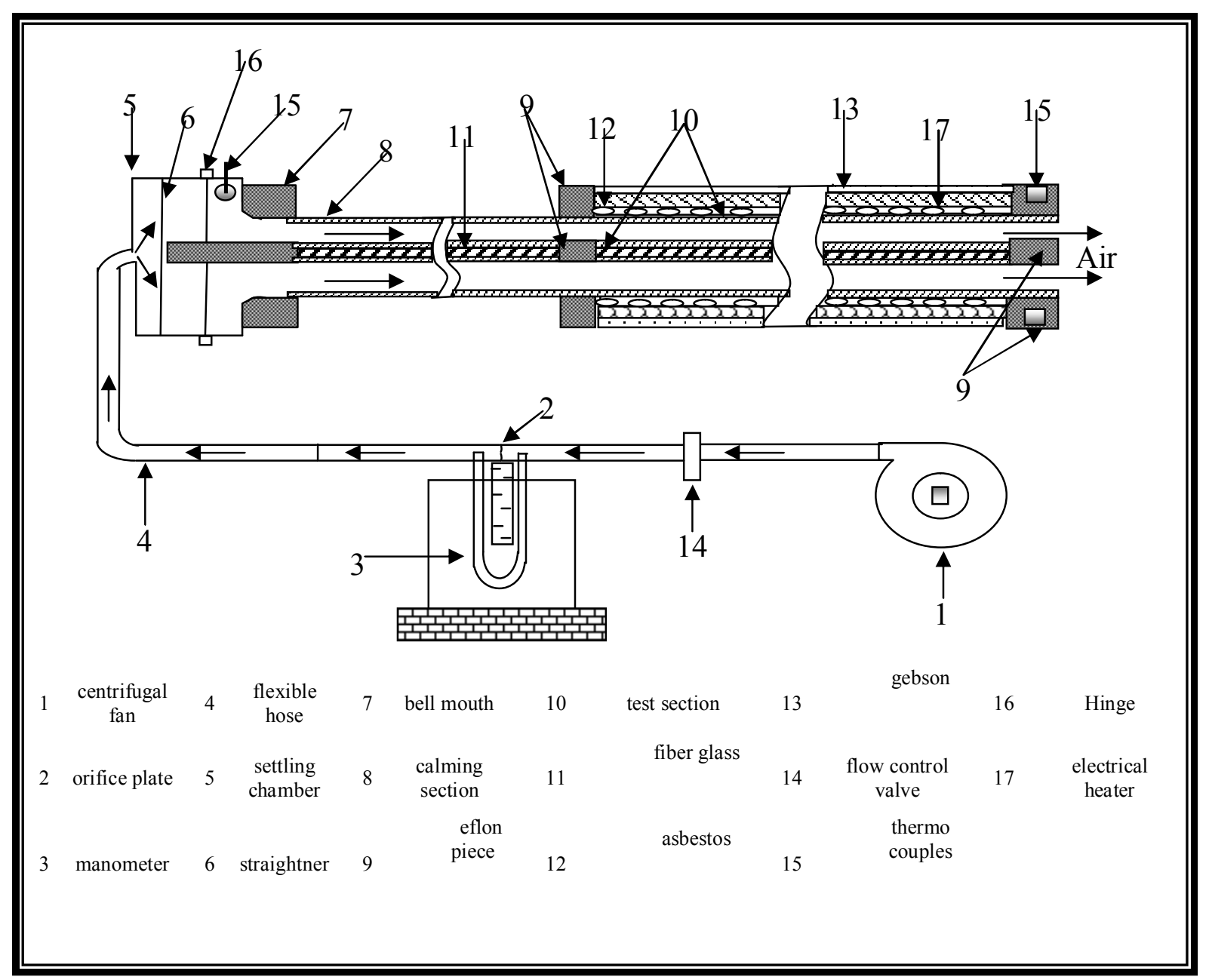

Fig .(1) Diagram of Experimental Arrangement 


\section{Uncertainty analysis:}

The accuracy of experimental results depends upon the accuracy of the individual measuring instruments and the manufacturing accuracy of the circular annular tube. The accuracy of any instrument also is limited by its minimum division (its sensitivity). The calculation of the error, precision, and the general validity of the experimental measurements were carried out. In fact, the magnitude of the experimental error is always uncertain. In the present work, the uncertainties in heat transfer coefficient (Nusselt number), Reynolds number and Rayligh number were estimated following the differential approximation method as reported by [Holman 1984]. The differential approximation method was considered to evaluate the uncertainty in result $\mathrm{R}$ that is a function of the independent parameter: $\mathrm{Y} 1, \mathrm{Y} 2$, Y3 ........ $Y_{n}$. i.e.

$\mathrm{R}=\mathrm{R}\left(\mathrm{Y}_{1}, \mathrm{Y}_{2}, \mathrm{Y}_{3} \ldots \ldots \mathrm{Y}_{\mathrm{n}}\right)$

At the same time it may perturb the variables by $\Delta \mathrm{Y}_{1}, \Delta \mathrm{Y}_{2}, \Delta \mathrm{Y}_{3}, \ldots . \Delta \mathrm{Y}_{\mathrm{n}}$, then:

$\mathrm{R}\left(\mathrm{Y}_{1}+\Delta \mathrm{Y}_{1}\right)=\mathrm{R}\left(\mathrm{Y}_{1}+\Delta \mathrm{Y}_{1}, \mathrm{Y}_{2}, \mathrm{Y}_{3}, \ldots \ldots . \mathrm{Y}_{\mathrm{n}}\right)$

$R\left(Y_{n}+\Delta Y_{n}\right)=R\left(Y_{1}+Y_{1}, Y_{2}, Y_{3}, \ldots \ldots . . Y_{n}+\Delta Y_{n}\right)$

Therefore, for small enough values of the quantities $\Delta \mathrm{Y}_{1}, \Delta \mathrm{Y}_{2}, \Delta \mathrm{Y}_{3}, \ldots \ldots . \Delta \mathrm{Y}_{\mathrm{n}}$, the partial derivatives can be well approximated by:

$\frac{\partial R}{\partial Y_{i}} \approx \frac{R\left(Y_{i}+\Delta Y_{i}\right)-R\left(Y_{i}\right)}{\Delta Y_{i}}$

where $\mathrm{i}=1,2,3, \ldots \ldots . . \mathrm{n}$

If there are uncertainties $\mathrm{W}_{1}, \mathrm{~W}_{2}, \mathrm{~W}_{3}, \ldots \ldots \mathrm{W}_{\mathrm{n}}$, in the independent variables and $\mathrm{W}_{\mathrm{R}}$, is the uncertainty in the result on the same odds, then the uncertainty in the result can be given as :

$W_{R}=\left[\left(\frac{\partial R}{\partial Y_{1}} W_{1}\right)^{2}+\left(\frac{\partial R}{\partial Y_{2}} W_{2}\right)^{2}+\left(\frac{\partial R}{\partial Y_{3}} W_{3}\right)^{2}+\ldots . .+\left(\frac{\partial R}{\partial Y_{n}} W_{n}\right)^{2}\right]^{\frac{1}{2}}$

Since the values of the partial derivative and the errors in the measuring parameters may be positive or negative, then the absolute values are considered to obtain the maximum absolute uncertainty in the result $\mathrm{W}_{\mathrm{R}}$. For a typical experiment, the total uncertain in measuring the heater input power, temperature difference $\left(\mathrm{T}_{\mathrm{w}}-\mathrm{T}_{\mathrm{b}}\right)$, the heat transfer rate, the circular outer tube surface area and the air flow rate were $\pm 0.2 \%, \pm 0.33 \%, \pm 1.8 \%, \pm 1.5 \%$, and $\pm 0.02 \%$, respectively. These were combined to give a maximum error of $\pm 1.45 \%$ in heat transfer coefficient (Nusselt number) and maximum error of $\pm 1.35 \%$ in Reynolds number and $\pm 1.41 \%$ in Rayliegh number. The error related to heat transfer coefficient seems to be acceptable since the acceptable error in heat transfer problems is about $10 \%$.

\section{Experimental procedure:}

The input electric power to the working annulus is regulated by an $\mathrm{AC}$ power variac and measured by a digital wattmeter with a resolution of $0.01 \mathrm{~W}$. the steady state is considered to be achieved when the temperature reading of each thermocouple did not change by more than $0.5{ }^{\circ} \mathrm{C}$ within $25 \mathrm{~min}$. When the steady state condition is established, the 
reading of all thermocouples, the input power and the inlet and outlet bulk temperatures are recorded. The readings of all thermocouples have been taken by a precalibrated digital temperature recorder capable of reading $0.01{ }^{\circ} \mathrm{C}$ via a multi-switch. The steady state condition for each run achieved after approximately 4 hours. In the present work, calming section (entrance section) has been used that in which the flow is hydrodynamically developed, thermally developing and thermally fully developed at the entrance of the test section in the present work, the Reynolds number under consideration is ranged from 450 to 2000, indeed this range has been selected after so many experimental attempts in order to ensure that the mixed convection regime has been covered and accordingly this range gives the thermally developing flow and the thermally fully developed flow cases.

\section{Checking the assumptions:}

Theoretically, to have a hydrodynamically developed flow, the hydrodynamic entry length of the flow is a function of Re for low Reynolds number flow as found by solving the complete set of Navier-Stokes Equation. [Shah and London 1978] in their book gave two equations for the hydrodynamic entry length as follows:

$$
\begin{aligned}
& \left(\frac{L_{h y}}{D_{h}}\right)=0.59+0.056 \mathrm{Re} \\
& \left(\frac{L_{h y}}{D_{h}}\right)=\frac{0.6}{0.035 \mathrm{Re}+1}+0.056 \mathrm{Re}
\end{aligned}
$$

The $\mathrm{L}_{\mathrm{hy}}$ predicted by Eq.(5) is somewhat higher than $\mathrm{L}_{\mathrm{hy}}$ observed experimentally, where the definitions of both $\mathrm{L}_{\mathrm{hy}}$ are the same (a dimensionless duct length required to achieve $\mathrm{u}_{\max }$ as $0.99 \mathrm{U}_{\text {max,fd}}$ ). So, Eq.(6) is in better agreement with the experimental values

\section{[Shah and London 1978].}

In the present work, the horizontal position has been chosen to carry out the experiments in order to cover the phenomena of hydrodynamic fully developed mixed convection in the thermal entrance region of annular tube because of importance this position in the practical applications and to give more flexibility for choosing the length of calming section so as to obtain high ranges of Reynolds number. Hence, the deduct the maximum Reynolds number that is used to carry out experiments, Eq.(6) can be written as follows:

$$
0.00196 \operatorname{Re}^{2}+\left(0.056-0.035 \frac{L_{h y}}{D_{h}}\right) \operatorname{Re}+\left(0.6-\frac{L_{h y}}{D_{h}}\right)=0
$$

But the higher value of hydrodynamic entry length has been chosen as $3 \mathrm{~m}$ (i.e,, $\mathrm{L}_{\mathrm{hy}} / \mathrm{D}_{\mathrm{h}}=100$ ), the equation (7) becomes:

$0.00196 \mathrm{Re}^{2}-3.444 \mathrm{Re}-99.4=0$

The solution of this quadratic equation gives the maximum value of Reynolds number as 1785 .The lower value of $L / D_{h}$ will certainly give lower value of $R e$.

Therefore, from a theoretical point of view, we should carry out the experiments from Reynolds number lower than 1785 , so that the flow may be hydrodynamically developed. But, is not enough for us. To avoid this constraint and to increase the maximum Reynolds number that we can use, a stabilization settling chamber of $300 \mathrm{~mm}$ length has been added at the beginning of calming section. Later on, having like purpose to check if the stabilization chamber helps us to have a hydrodynamically developed flow even for Reynolds number 
higher than 1785, we carried out experiments for the Reynolds number range from 450 to 2000 (i.e; the case of hydrodynamic fully developed will happen only at $L / D_{h}=100$ ).

\section{RESULTS AND DISCUSSION}

Generally, the temperature and the local Nusselt number distributions along the longitudinal axis for the case of mixed convection circular duct (cylindrical or annular) have the same shape shown in the work of (Abdulhasan, et al) .In the present work, the effect of heat flux, Reynolds number, and as a result Richardson number on the average temperature and Nusselt number at the outer heated tube of an annulus for a selected run is carefully studied.

Fig.(2\&3) show the variation of outer tube average temperature and Nusselt number with the heat flux ;respectively, for various Reynolds number. The following remarks can be noticed from this figure as follows:

1- For all values of Reynolds number the average temperature value increases as heat flux increases and reaches the value of $800 \mathrm{~W} / \mathrm{m}^{2}$ (critical value)then the behavior is reversed and vice versa for the average Nusselt number. This behavior can be attributed to that when the heat flux lies between $(200-800) \mathrm{W} / \mathrm{m}^{2}$ the secondary currents resulted from buoyancy force is weak, as a result the values of average temperature increase and the values of average Nusselt number decrease as shown in Fig.(3). Beyond $\mathrm{q}=800 \mathrm{~W} / \mathrm{m}^{2}$ (critical value), the secondary currents will be stronger, that leads to decreasing the average temperature values and increasing the heat transfer coefficients which are represented by $\mathrm{Nu}_{\mathrm{m}}$.

2- At constant heat flux, the value of average temperature increases as Reynolds number decreases and rice versa for averages Nusselt number because the dominated forced convection in the heat transfer process.

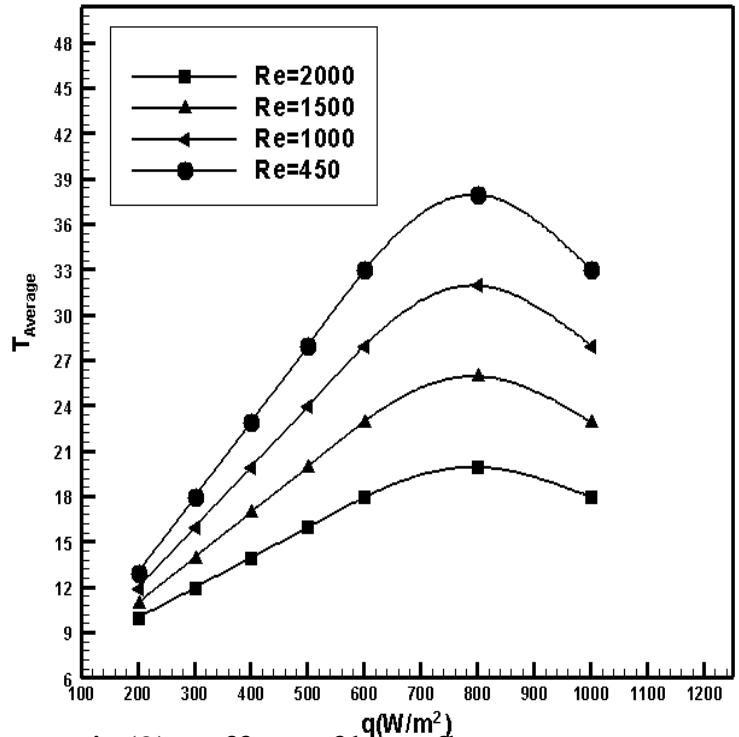

Fig(2): Effect of heat flux on average temperature of outer tube for various Reynolds number

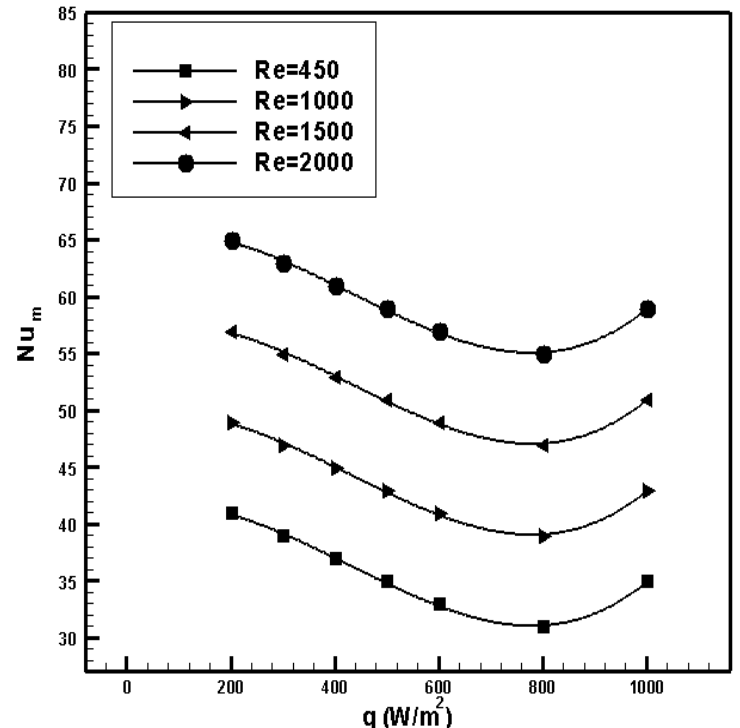

Fig(3): Effect of heat flux on average

Nusselt number of outer tube for various Reynolds number

Fig.(4\&5) show the effect of Richardson number on average temperature and Nusselt number of outer tube for various Rayliegh number $\left(\mathrm{Ra}=4.5 \times 10^{4}, 5.3 \times 10^{4}, 5.8 \times 10^{4}\right.$ and $6.8 \times 10^{4}$ ). as be shown in Fig.3 for the same Rayliegh number the average temperature 
increases with increasing of Richardson number until Ri $\approx 0.6$ (critical values) then the behavior is reverse and vice versa for the average Nusselt number as shown in Fig.(5). This can be attributed to that at low values of Richarson number $\mathrm{Ri}<0.6$ the effect of Reynolds number (forced convection) is greater of Rayliegh number (natural convection), as result the secondary current will be weak leads to reducing in the heat transfer coefficients, but at high values of Richardson number $\mathrm{Ri}>0.6$, the natural convection will be the dominating factor in the heat transfer process. Which improve as $\mathrm{Ri}$ increases. There is not doubt that, the secondary flow due to free convection appears at the start of the annular duct entrance region. This secondary velocity is initially higher in the upper region of the annulus, and increases throughout the cross section until its intensity reaches a maximum at a certain downstream position which depends on Rayliegh number. Further downstream, the secondary flow diminishes gradually, and ultimately vanishes altogether. Increasing Rayliegh number decreases the thermal entrance length prior to the onset of marked free convection effects (i.e; the average nusselt number increases).

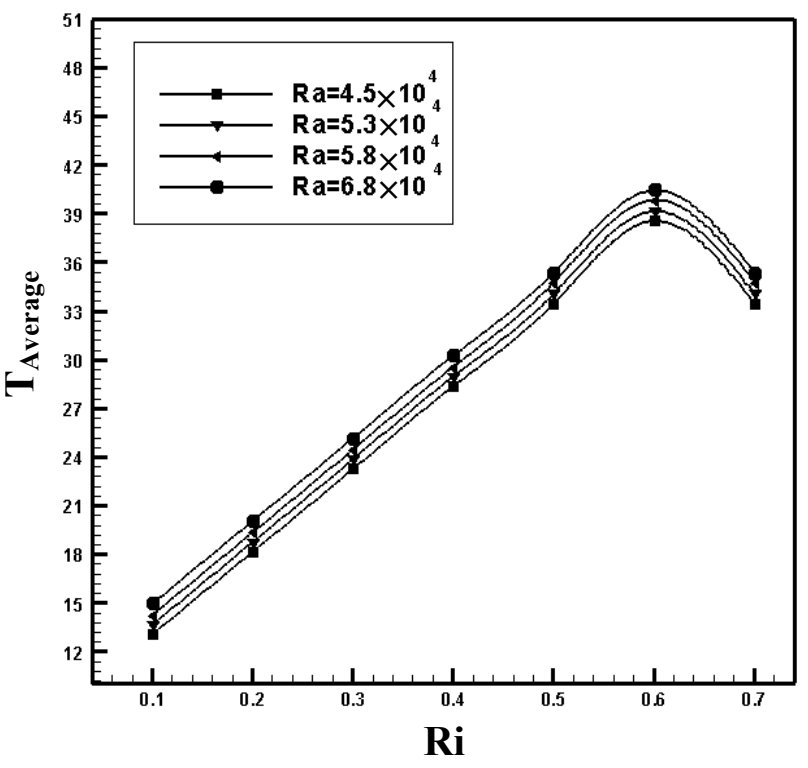

Fig(4): Effect of Richarson number on average temperature of outer tube for various Rayliegh number

The effect of hydrodynamic development length $\left(\mathrm{L} / \mathrm{D}_{\mathrm{h}}\right)$ on the average Nusselt number for various Reynolds number and for $\mathrm{Ra}=5.6 \times 10^{4}$ is shown in Fig.(6). As can be seen from this figure that the heat transfer process improves as $\left(\mathrm{L} / \mathrm{D}_{\mathrm{h}}\right)$ increases since as the annular duct is long enough, the velocity will be eventually fully developed in which the fluid temperature in the test section approaches the temperation of heated wall at some where region leading to accelerate the thermal boundary layer development and improves the heat transfer process.

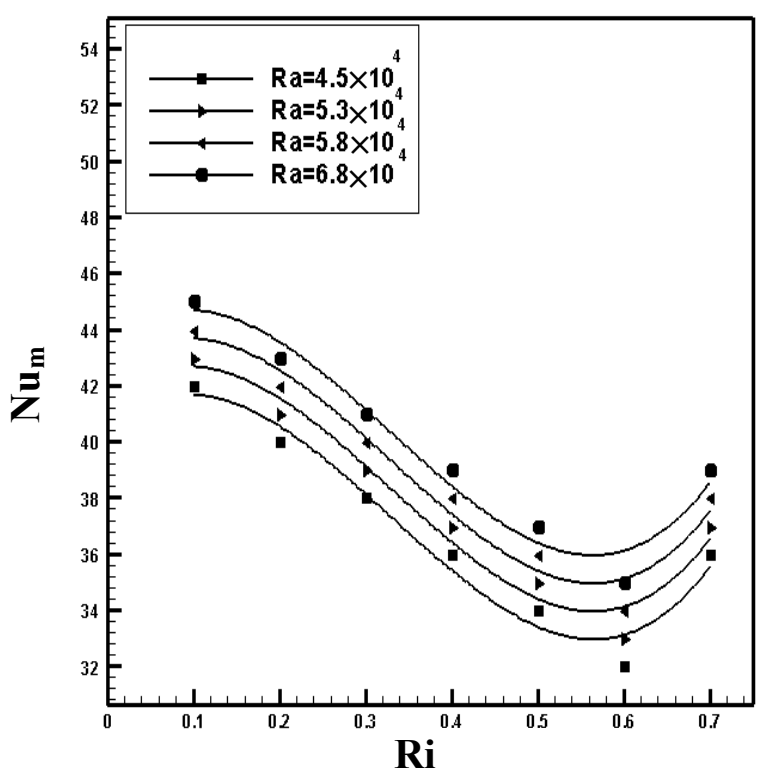

Fig(5): Effect of Richarson number on

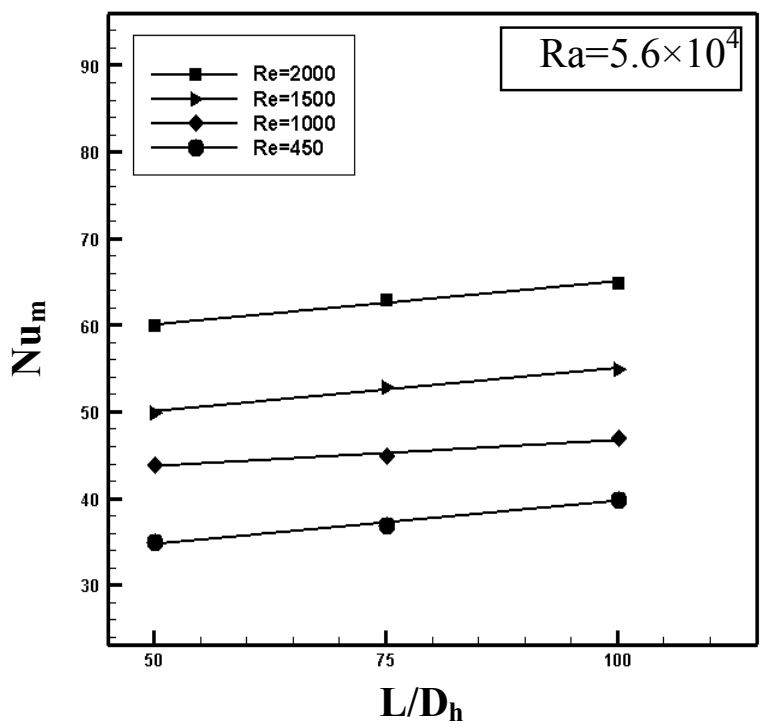

Fig(6): Effect of $L / D_{h}$ on $\mathrm{Nu}_{\mathrm{m}}$ for different values of Reynolds number 
The circumferential variations of the local Nusselt number at three positions along the outer tube $(\mathrm{x}=0.2 \mathrm{~m}, \quad 0.6 \mathrm{~m}, \quad$ and $1.2 \mathrm{~m})$ for $\left(\mathrm{Ra}=4.5 \times 10^{4} \& \mathrm{Re}=450\right)$ and $\left(\mathrm{Ra}=6.8 \times 10^{4} \& \mathrm{Re}=2000\right)$ are shown in Figs. $(7 \& 8)$;respectively. As can be shown in Fig. 6 the maximum local Nusselt number along the outer tube length occurs approximately between $\phi=120^{\circ} \& 160^{\circ}$. It is noticed also that the values of circumferential Nusselt number decrease as the flow moves further downstream in the region between $\phi=0^{\circ} \& 141^{\circ}$ which the values are the same. Beyond this angular position, the behavior reverses completely. In general, since colder fluid tends to collect below the inner tube and hotter fluid above it, large temperature gradients and higher local Nusselt numbers are to be expected a the bottom. The secondary flows associated with free convection behave so as to reduce temperature difference in the annulus. In the case of outer wall heated, the associated vortex flows have their center in the lower annulus. The vortex strength increases as Rayliegh number and Reynolds number increases as shown in $\operatorname{Fig}(8)$ in which the values of circumferential Nusselt number are higher than that in Fig.(7) and increase linearly with the angular position $(\phi)$ and tend to be equal along the outer tube length between $\phi=0^{\circ} \& 90^{\circ}$, then they increase with flow direction.

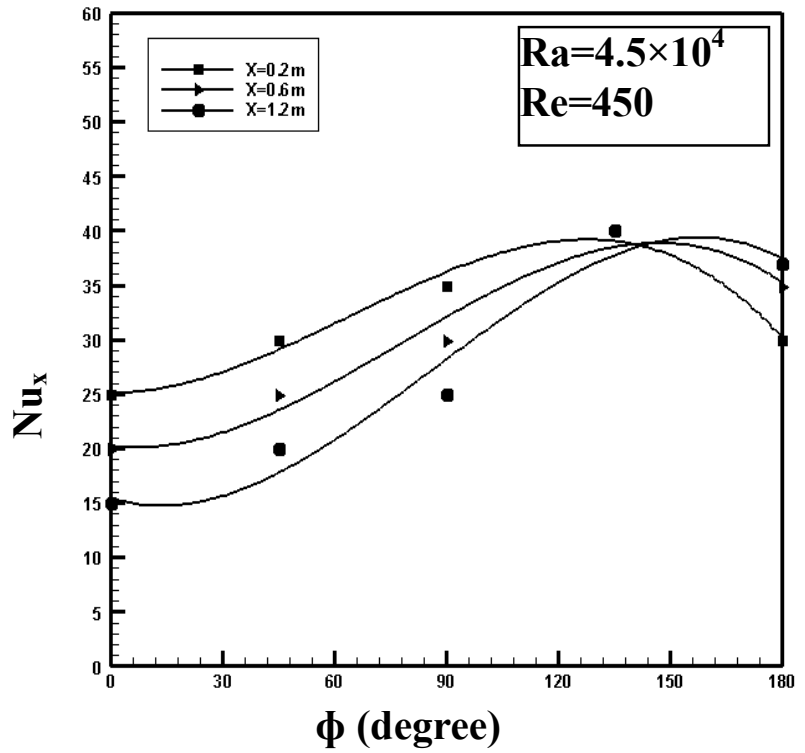

Fig(7): Variation of local Nusselt number with the angular position at three longitudinal position with $\mathrm{Re}=450$ and $\mathrm{Ra}=4.5 \times 10^{4}$

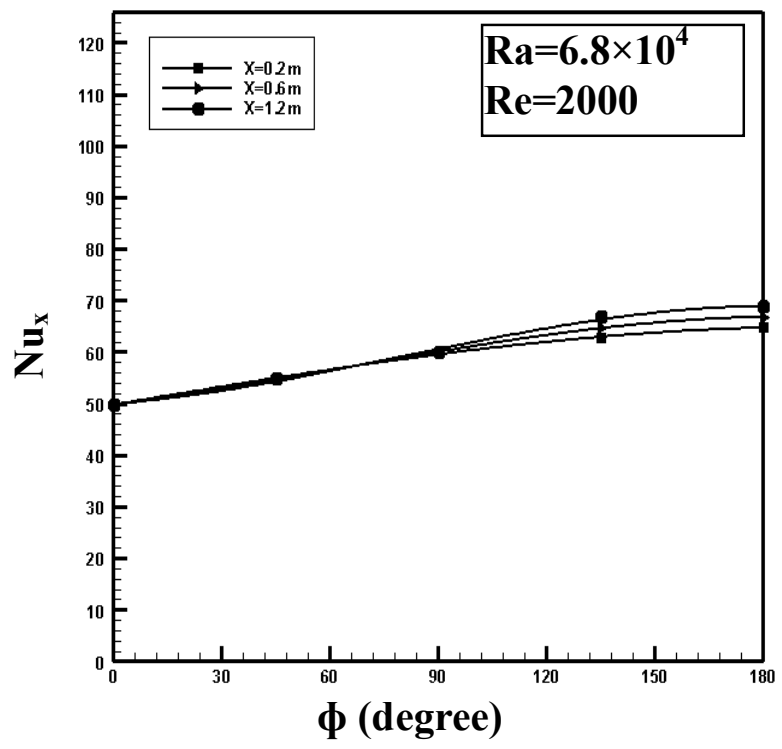

Fig(8): Variation of local Nusselt number with the angular position at three longitudinal position with $\mathrm{Re}=2000$ and $\mathrm{Ra}=6.8 \times 10^{4}$

\section{Average heat transfer correlations:}

Since, the heat transfer process in the horizontal annulus with uniformly heated outer tube improves as the length of development section increases, the results of average Nusselt number one correlated in empirical equation and are plotted in Fig(9) in from of $\log$ $\mathrm{Nu}_{\mathrm{m}}\left(\mathrm{L} / \mathrm{D}_{\mathrm{h}}\right)_{\text {cal }}$ against $\log \mathrm{Ra} / \mathrm{Re}$. as can seen from this figure that all the points are represented by straight line of the following equation:

$$
\mathrm{Nu}_{\mathrm{m}}=4.99(\mathrm{Ra} / \mathrm{Re})^{-0.569}\left(\mathrm{~L} / \mathrm{D}_{\mathrm{h}}\right)_{\mathrm{cal}}^{-0.0154}
$$


Fig(10) shows the relation between $\mathrm{Nu}_{\mathrm{m}} / \operatorname{Re}\left(\mathrm{L} / \mathrm{D}_{\mathrm{h}}\right)$ against Rayliegh number for the present work and (Ciampia) work to gives the same trend and behavior.

The results of Ciampia work for the average Nusselt number were correlated in the following empirical equation:

$$
\mathrm{Nu}_{\mathrm{m}}=0.2115(\mathrm{Ra})^{0.34}(\mathrm{Re})^{0.08}\left(\mathrm{~L} / \mathrm{D}_{\mathrm{h}}\right)^{-0.0113}
$$

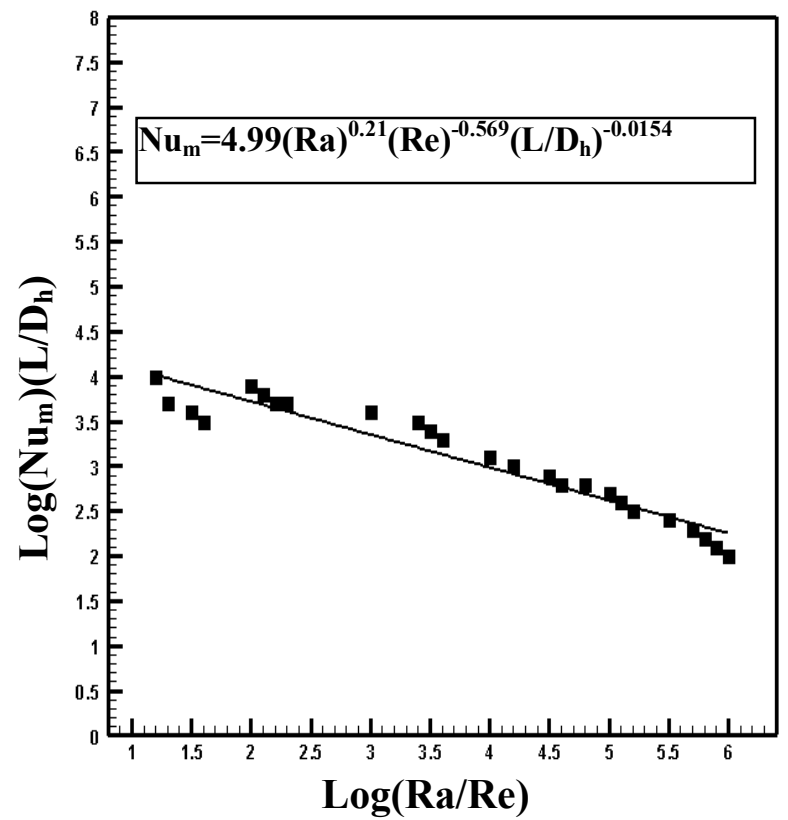

Fig(9): Correlation of average heat transfer data

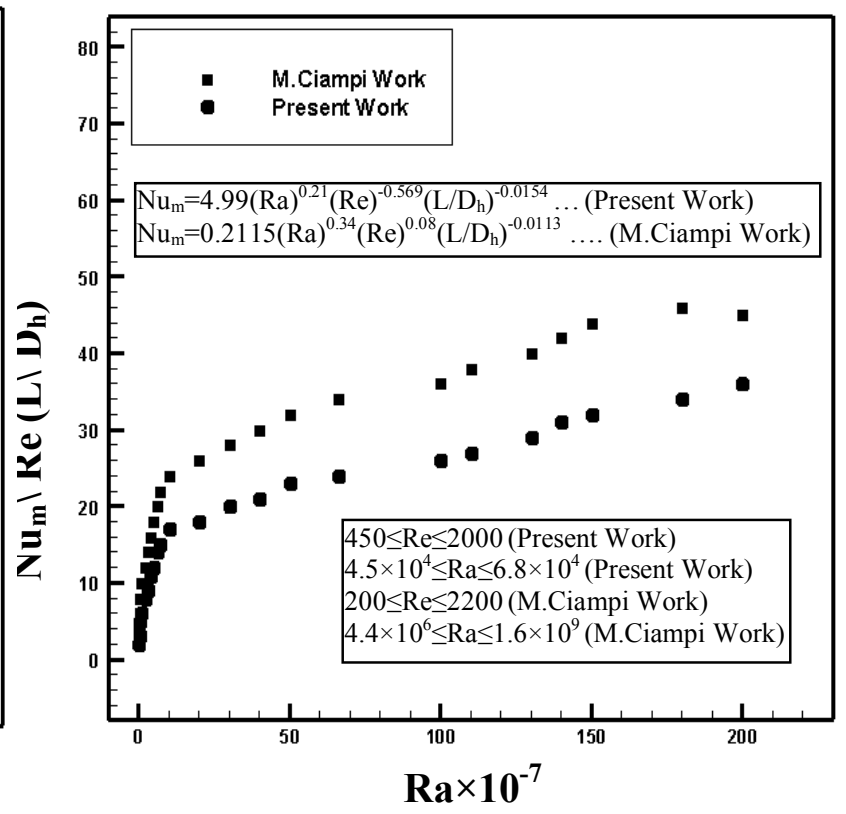

Fig(10): Comparison the present work with Ciampi work for $\mathrm{Nu}_{\mathrm{m}} / \mathrm{Re}\left(\mathrm{L} / \mathrm{D}_{\mathrm{h}}\right)$

\section{CONCLUSIONS}

1- At axial locations where the secondary motion is prominent, the local Nusselt number becomes higher below the horizontal axis than above the horizontal axis.

2- The entrance section length has important role in the heat transfer process which improves as the flow becomes completely fully developed.

3- The physical behavior of the heat transfer process by mixed convection phenomena depends greatly on Richardson number. This number has a critical value beyond it, the physical behavior is reversed. In this research, for $\mathrm{Ri}>0.6$, the heat transfer process improves because of the dominating natural convection which will be stronger than the forced convection.

4- Increasing Rayliegh number decreases the thermal entrance length prior to the onset of marked natural convection effects.

5- The average Nusselt number data are correlated with $\mathrm{Ra}, \mathrm{Re}$, and $\left(\mathrm{L} / \mathrm{D}_{\mathrm{h}}\right)_{\mathrm{cal}}$, and agreed with the results of (Ciampi) work. 


\section{NOMENCLATURE}

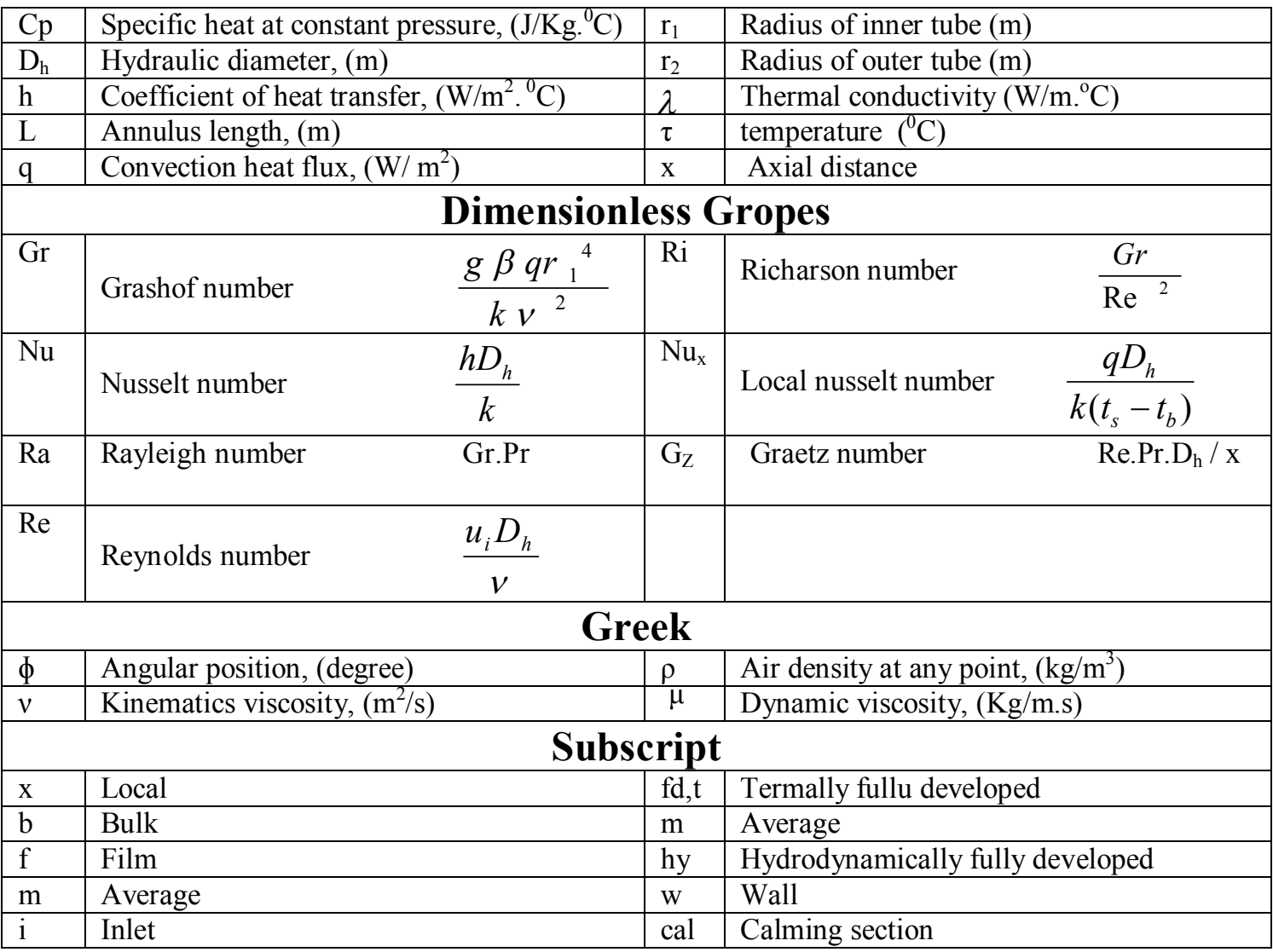

\section{REFERENCES}

\section{[Abdulhassan A. K. ,et-al]}

"Buoyancy Effects On Opposing Laminar Flow In A Uniformly Heated Inclined Cylinder" The Iraqi Journal for Mechanical and Material Eengineering (Babylon University, special issue (a) PP.26-40 (2009).

\section{[Cimpi M.,Faggiani S.,Grassai W. and Tuoni G.]}

"Mixed convection heat transfer in horizontal, concentric annuli for transitional flow conditions" in Heat Mass Transfer, Vol.30, No.5.PP.883-841 (1987).

\section{[Gada A. Sadiq]}

"Developing laminar mixed convection heat transfer through concentric annuli with adiabatic inner cylinder" M.SC. Thesis, University of Baghdad, College of Engineering, Mars (2009).

\section{[J. P. Holman]}

"Experimental Method for Engineers", McGraw-Hill,Tokyo,Japan,4 $4^{\text {th }}$ Edition (1984)

[Habibm.A. and Negm A.A.]

"laminar mixed convection in horizontal concentric annuli with non-uniform circumferential heating" J.Heat and Mass Transfer, Vol.37,PP.427-435, Numbers 4-5, July,(2001).

\section{[Kaviaany M.]}


"Laminar combined convection in a horizontal annulus subjected to constant heat flux inner wall and adiabatic outer wall" J.Heat Transfer,Vol.108, PP. 392-397, May,(1986).

\section{[Kotake S. and Hattori N.]}

"Combined forced and free convection heat transfer for fully-developed laminar flow in horizontal annuli " Int J. Heat Mass Transfer Vol.28, No. 11, PP.2133-2120,(1985).

\section{[Mohammed. A. R. Nima]}

"Developing laminar mixed convection heat transfer through concentric annuli" M.sc. Thesis, University of Baghdad, College of Engineering Mechanical Department, (2007).

\section{[M.Ciampi,et-al]}

"experimental study of mixed convection in horizontal annuli for low Rynolds numbers"Scool of Mechanical Engineering, Purdue University, Indiana, U.S.A. PP.14131416.

\section{[Nazrul Islam,Gaitonde U. N. and Sharma G.K.]}

"Mixed convection heat transfer in the entrance region of horizontal annuli" Int. J. Heat Mass Transfer. Vol.44, No.11,PP.2107-2120, (2001).

[Nieckele A. O. and Patankar S. V.]

"laminar mixed convection in a concentric annulus with horizontal axis"J. Heat Transfer, Vol.107,PP.902-909, November, (1985).

[Shah R.K. and A. L. London]

"Laminar flow forced convection in duct" Advance in Heat Transfer, Academic Press, Newyork, Ch V, PP.98-99 (1978).

\section{The work was carried out at the college of Engineering. University of Technology}

